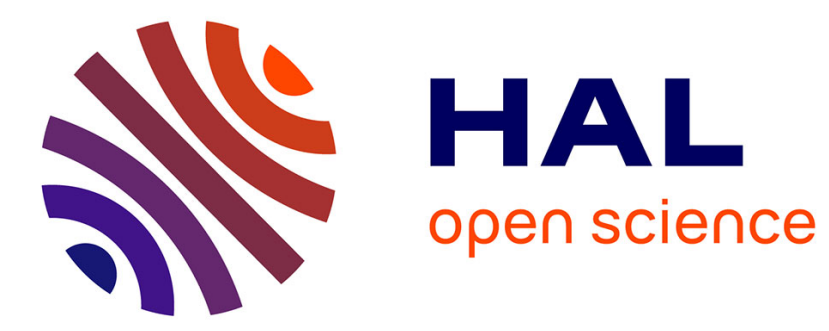

\title{
Fluorescent Biosensor of CDK5 Kinase Activity in Glioblastoma Cell Extracts and Living Cells
}

Marion Peyressatre, Arthur Laure, Morgan Pellerano, Hassan Boukhaddaoui, Ines Soussi, May C Morris

\section{- To cite this version:}

Marion Peyressatre, Arthur Laure, Morgan Pellerano, Hassan Boukhaddaoui, Ines Soussi, et al.. Fluorescent Biosensor of CDK5 Kinase Activity in Glioblastoma Cell Extracts and Living Cells. Biotechnology Journal, 2020, 15 (9), pp.1900474. 10.1002/biot.201900474 . hal-03004023

\section{HAL Id: hal-03004023 \\ https://hal.science/hal-03004023}

Submitted on 13 Nov 2020

HAL is a multi-disciplinary open access archive for the deposit and dissemination of scientific research documents, whether they are published or not. The documents may come from teaching and research institutions in France or abroad, or from public or private research centers.
L'archive ouverte pluridisciplinaire HAL, est destinée au dépôt et à la diffusion de documents scientifiques de niveau recherche, publiés ou non, émanant des établissements d'enseignement et de recherche français ou étrangers, des laboratoires publics ou privés. 


\title{
Fluorescent biosensor of CDK5 kinase activity in glioblastoma cell extracts and living cells
}

\author{
Marion Peyressatre $^{1}$, Arthur Laure ${ }^{1}$, Morgan Pellerano ${ }^{1}$, Hassan Boukhaddaoui ${ }^{2}$, Ines Soussi ${ }^{1}$ \\ \& May C. Morris ${ }^{1}$ \\ ${ }^{1}$ Institut des Biomolécules Max Mousseron, CNRS, UMR 5247, Université de Montpellier \\ Faculté de Pharmacie, 15, Av. Charles Flahault, 34093 Montpellier, France \\ ${ }^{2}$ Plateau Imagerie Cellulaire MRI-INM, INM-INSERM U 1051 \\ Hôpital Saint Eloi, 80 rue Augustin Fliche, 34091 Montpellier, France
}

Running title: Fluorescent reporter of CDK5 activity

Correspondence: Dr. May C. Morris. Institut des Biomolécules Max Mousseron, CNRS, UMR 5247, Université de Montpellier, Faculté de Pharmacie, 15, Av. Charles Flahault, 34093 Montpellier, France. Phone: +34 (0) 411759624 ; Email: may.morris@umontpellier.fr

Keywords: CDK5; Fluorescent Biosensor; Glioblastoma; Kinase activity; Peptide Abbreviations: CDK: Cyclin-dependent Kinase ; PAABD : phospho-amino acid binding domain 


\begin{abstract}
CDK5 plays a major role in neuronal functions, and is hyperactivated in neurodegenerative pathologies as well as in glioblastoma and neuroblastoma. Although this kinase constitutes an established biomarker and pharmacological target, there are few means of probing its activity in cell extracts or in living cells. To this aim we engineered a fluorescent peptide reporter of CDK5 kinase activity, derived from a library of CDK5-specific substrates, and established its ability to respond to recombinant $\mathrm{CDK} 5 / \mathrm{p} 25$ and report on CDK5 activity in glioblastoma cell extracts through sensitive changes in fluorescence intensity. We further developed a cell-penetrating variant of this biosensor which can be implemented to image CDK5 activation dynamics in space and in time. This original biosensor constitutes a potent tool for quantifying differences in CDK5 activity following treatment with selective inhibitors and for monitoring CDK5 activation, following inhibition or stimulation, in a physiologically-relevant environment. As such it offers attractive opportunities to develop a diagnostic assay for neuronal pathologies associated with hyperactivated CDK5, as well as a companion assay to evaluate response to new therapies targeting this kinase.
\end{abstract}

\title{
Novelty Statement
}

To address the lack of technologies available to probe and quantify CDK5 activity, we have engineered a fluorescent peptide reporter that reports on CDK5 activity in vitro and a self-penetrating derivative that enables imaging of CDK5 activation in living cells. This original biosensor constitutes a potent tool for quantifying differences in CDK5 activity following treatment with selective inhibitors and for monitoring CDK5 activation, following inhibition or stimulation, in a physiologically-relevant environment. As such it offers attractive opportunities to develop a diagnostic assay for neuronal pathologies associated with hyperactivated CDK5, as well as a companion assay to evaluate response to new therapies targeting this kinase. 


\section{Introduction}

Glioblastoma is an aggressive malignant primary brain tumor that ranks amongst the most lethal of all human cancers. Current treatment involves chemotherapy with temozolomide and radiation, but only offers palliation [1]. Moreover, prediction of the onset of this disease is limited by an overall lack of specific biomarkers. One of the major challenges to improve early diagnostics and associated development of targeted therapies therefore resides in the identification of relevant biomarkers and development of sensitive diagnostic strategies.

Over the past recent years, the serine/threonine protein kinase CDK5 has emerged as an attractive biomarker for monitoring the onset and development of glioblastoma. This $34 \mathrm{kDa}$ neurospecific serine/threonine protein kinase and unconventional member of the CDK family, initially identified through structural homology with CDK2, and independently isolated as a proline-directed histone $\mathrm{H} 1$ kinase from bovine brain, plays a central role in regulation of an ensemble of neuronal functions, including neurite extension and neuronal migration, synapse formation during brain development, synaptic plasticity, axonal guidance and synaptic transmission activities in mature neurons [2-12] CDK5 is essential during early neurodevelopment in mammals, and mice lacking CDK5 exhibit severe defects in the layering of the cerebral cortex [11-13]. The importance of CDK5 in neuronal functions is underscored by the impact of its dysregulation, CDK5 hyperactivation contributing to several neurodegenerative diseases, including Alzheimer's, Parkinson's, Huntington's and amyotrophic lateral sclerosis [14-16]. More recent studies have highlighted the implication of CDK5 in glioblastoma, where it has been reported to be overexpressed and hyperactive and CDK5 has been identified as a valuable predictive marker of tumorigenesis and progression in glioma [17-19]. Specifically, increased levels of CDK5 lead to hyperphosphorylation of Isoform A of phosphatidylinositol 3-kinase enhancer (PIKE-A) in human glioblastoma specimens, thereby stimulating PIKE-A GTPase activity, which activates nuclear Akt and promotes growth factorinduced migration and invasion of human glioblastoma cells [20]. CDK5 has been found to phosphorylate dynamin-related protein 1 (DRP1), thereby stimulating its activity in brain tumourinitiating cells ; DRP1 activation correlates with poor prognosis in glioblastoma [21].

CDK5 is associated with its neurospecific partner p35, which anchors it at the membrane through a myristoylation site [22, 23]. Following an influx of calcium, p35 undergoes calpain-mediated cleavage into $\mathrm{p} 25$, which leads to release of the CDK5/p25 complex into the cytoplasm, where it phosphorylates a variety of substrates, the most notorious of which is the microtubule-associated protein Tau $[24,25]$. Hence it is assumed that CDK5 is primarily active in the cytoplasm. However despite the physiological and pathological relevance of this neurospecific kinase, its molecular 
mechanism of activation, and in particular its spatio-temporal dynamics remain to be investigated. There are currently no tools available to determine the relative activity of CDK5 in a complex biological environment or to monitor its dynamics in living cells in real-time. To this aim we have developed a CDK5-specific fluorescent peptide biosensor (CDKACT5) that reports on CDK5 kinase activity through sensitive changes in fluorescence intensity. In this study we describe the design and characterization of this biosensor derived from a peptide library of CDK5 substrates. We show that it responds to recombinant CDK5/p25 and can report on CDK5 activity in U87 glioblastoma cell extracts in a dose-dependent fashion, thereby enabling quantification of differences in kinase activity upon stimulation or treatment with ATP-competitive inhibitors in a complex and physiologicallyrelevant environment. We have further developed a self-cell-penetrating variant of this CDK5 biosensor and show that it can be implemented to probe CDK5 activity in real-time in living glioblastoma cells by fluorescence imaging, thereby providing means to quantify the relative activity of CDK5 following treatment with inhibitors, and to monitor the dynamics of its activation in space and in time.

\section{Materials and Methods}

\subsection{Design and synthesis of CDKACT5 peptide biosensor library.}

CDKACT5 biosensors were designed to combine a sequence derived from the WW domain of Pin 1 (sequence GFARVYMSRSSGWERPSGG described in [26]) with a substrate sequence derived from reported substrates of CDK5 into which a unique cysteine was introduced at position -2 relative to the serine or threonine of the S/T-P phosphorylation site. A control peptide named CDKACT-Ctrl was designed with an irrelevant substrate domain derived from ubiquitin [28]. A self-cell-penetrating variant of CDKACT5-ER104 was designed by adding the first 13 residues of the cell-penetrating peptide Pep1 [27] to the N-terminus of CDKACT5-ER104, and a nonphosphorylatable mutant was made by substituting the serine phosphorylation site by an alanine. Peptides were synthesized by GLS Biochem and purified to $95 \%$ by HPLC and LCMS was performed by GLS Biochem. They were labelled with a 10-fold molar excess of Cy5-maleimide, then further purified on NAP5 columns in $50 \mathrm{mM}$ TrisHCl, pH 7.4, $150 \mathrm{mM} \mathrm{NaCl}$, to remove excess label. The list of CDKACT5 peptides used in this study are summarized below with the Cysteine used for labelling in bold and the phosphorylation site, substituted by an alanine in mutants, in red.

$\begin{array}{lll}\text { CDKACT5-Tau } & \text { GFARVYMSRSSGWERPSGG } & \text { SGYSSPGSCSTPGSR } \\ \text { CDKACT5-Tau2 } & \text { GFARVYMSRSSGWERPSGG } & \text { KSPVVSGDCSSPR } \\ \text { CDKACT5-ATM } & \text { GFARVYMSRSSGWERPSGG } & \text { TKKCSSPNKIAS } \\ \text { CDKACT5-RKIP } & \text { GFARVYMSRSSGWERPSGG } & \text { LGKCSTPTQVK }\end{array}$




$\begin{array}{lll}\text { CDKACT5-DARPP } & \text { GFARVYMSRSSGWERPSGG NPCSYTPPSLKAVQRIA } \\ \text { CDKACT5-SYN } & \text { GFARVYMSRSSGWERPSGG RPPACSSPQRQA } \\ \text { CDKACT5-ER104 } & \text { GFARVYMSRSSGWERPSGG LNSVCSSPLML } \\ \text { CDKACT5-ER118 } & \text { GFARVYMSRSSGWERPSGG } & \text { PQCSSPFLQPH } \\ \text { CDKACT-Ctr1 } & \text { GFARVYMSRSSGWERPSGG VESSDTIDNVKSKIQDKEGC }\end{array}$

\section{Self-penetrating CDKACT5-ER104 peptide biosensors}

PepCDKACT5-ER104 KETWWETWWTEKK GFARVYMSRSSGWERPSGG LNSVCSSPLML PePCDKACT5-ER104mut KETWWETWWTEKK GFARVYMSRSSGWERPSGG LNSVCSAPLML

\subsection{Protein expression and purification of Cyclin-dependent Kinases.}

Recombinant GST-CDK5, GST-CDK2 and GST-CIV, the CDK-activating kinase of Saccharomyces cerevisiae, were expressed in E. coli by IPTG induction and purified by standard affinity and gel filtration chromatography as described previously [29, 30]. 6His-p25 and 6His-Cyclin A were expressed in E. coli by IPTG induction and purified on Ni-NTA resin [29-31]. GST-CDK5 / 6Hisp25 complex was formed following individual purification of each subunit and stoichiometric incubation, then further incubated with GST-CIV to (ensure complete activation). GST-CDK2 / 6HisCyclinA complex was formed by incubating 6His-CyclinA with GST-CDK2 on the GST affinity column, prior to elution.

\subsection{Fluorescence kinase assays.}

Fluorescence kinase assays were performed in 96-well plates in a thermostated chamber (Clariostar spectrofluorimeter, BMG) at $30^{\circ} \mathrm{C}$ in $200 \mu \mathrm{L}$ phosphate buffer saline (PBS) purchased from Sigma, supplemented with $5 \mathrm{mM} \mathrm{MgCl}_{2}, 0.5 \mathrm{mM}$ ATP, using $200 \mathrm{nM}$ Cy5-labelled peptide biosensors, except when stated otherwise. Changes in Cy5 fluorescence emission were recorded at $680 \mathrm{~nm}$ following excitation at $620 \mathrm{~nm}$. In all experiments, relative fluorescence was calculated following subtraction of CDKACT5-Cy5 fluorescence from fluorescence values obtained in the presence of protein kinases, cell extracts or inhibitors. Data analysis was performed using the GraFit Software (Erathicus Ltd). Experiments were performed in triplicate, and error bars indicate standard deviation from average. Histograms represent relative fluorescence intensity values after 2300s, unless specified otherwise.

\subsection{Protein Kinase Inhibitors}

Roscovitine, Dinaciclib (SCH727965), AT7519, U0126, PP2 and PD-0332991 were purchased from Euromedex. RO3306 was purchased from CalbioChem. Stock solutions were prepared at $10 \mathrm{mM}$ in DMSO and were freshly diluted in PBS to the desired concentration prior to use. 


\subsection{Cell culture and extract preparation.}

Cell culture media, serum and antibiotics were purchased from Life Technologies. PC12 and U87 cell lines used in this study were cultured in DMEM + Glutamax (Invitrogen) supplemented with $10 \%$ FCS, 100 units $/ \mathrm{mL}$ penicillin ( $\mathrm{G}$ sodium salt) and $100 \mu \mathrm{g} / \mathrm{mL}$ streptomycin at $37^{\circ} \mathrm{C}$ in an atmosphere containing $5 \% \mathrm{CO}_{2}$. Unsynchronized cells cultured to subconfluency were treated with either $20 \mu \mathrm{M}$ of retinoic acid (Sigma-Aldrich) or $10 \mu \mathrm{M}$ of ionomycin (Merck) for 90min to upregulate CDK5 activity. Alternatively cells were starved for $24-48 \mathrm{~h}$ by replacing medium supplemented with serum by DMEM devoid of serum. Cell extracts were prepared in PBS lysis buffer (PBS Sigma, 0.2\% NP40) complemented with $1 \mathrm{mM}$ EDTA, 2 mM PMSF, Complete ${ }^{\mathrm{TM}}$ protease inhibitors (Roche)), and total protein concentration was determined and normalized by spectrophotometric dosage at $280 \mathrm{~nm}$.

\subsection{SDS-PAGE and Western Blotting}

SDS-PAGE was performed according to standard protocols and proteins were transferred onto PVDF membranes (Immobilon-P, Merck Millipore) for $2 \mathrm{~h}$ at $100 \mathrm{~V}$ in precooled Towbin buffer using a liquid BioRad system. Western blotting was performed according to standard procedures : membranes were first saturated with 5\% skimmed milk in PBS, $0.1 \%$ Tween, then incubated overnight at $4{ }^{\circ} \mathrm{C}$ with the monoclonal mouse anti-CDK5 (Santa Cruz DC17 sc-249), actin antibody (Sigma-Aldrich A4700) or the monoclonal rabbit p35/p25 antibody (Cell Signaling C64B10) diluted 1:250 in PBS $0.1 \%$ Tween, then washed twice for $15 \mathrm{~min}$ with PBS $0.1 \%$ Tween and incubated for $1 \mathrm{~h}$ at room temperature with horseradish peroxidase-conjugated secondary antibodies (GE Healthcare) diluted 1:10000 in PBS 0.1\% Tween. After three final 5 minute washes with PBS 0.1\% Tween membranes were processed with ECL (GE Healthcare) for signal detection. Protein levels were determined by quantifying pixel intensity for each band of interest relative to pixel intensity of the actin band in the same sample.

\subsection{Cellular Internalization, Indirect Immunofluorescence and Fluorescence Microscopy.}

The self-cell-penetrating variant of CDKACT5-ER104 (Pep-CDKACT5-Cy5) and its mutant derivative (Pep-CDKACT5-ER104mut-Cy5) were incubated with a 2fold molar excess of Pep1 to optimize homogeneous intracellular delivery, then overlaid onto cultured U87 cells grown to 50-60 $\%$ confluency for $45 \mathrm{~min}$, essentially as described previously for the cell-penetrating peptide Pep1 [28]. Cellular internalization was complete after $45 \mathrm{~min}-1 \mathrm{hr}$, but cells were imaged after 3 -5hours to enable complete and homogeneous distribution of the fluorescent biosensor throughout cells and minimize aggregates. Pep1-Cy5 was similarly overlaid onto U87 cells grown to 50-60 \% confluency 
for 45min, and imaged 1-2 hours later. Cells were then extensively washed with PBS and either fixed with formalin/PBS for 5-10 min and processed for quantification of CDKACT5 activity, for indirect immunofluorescence, or returned to the incubator in DMEM supplemented with serum and antibiotics for time-lapse imaging. Quantification of CDK5 activity was performed by determining the fluorescence emission ratio of CDKACT5-Cy5/Hoechst in regions of interest (ROI) comprising three or four cells, using the ImageJ software. Background fluorescence was then subtracted from all images and the average fluorescence ratio of the Cy5-labelled biosensor over the Hoechst staining of nuclei in three different fields.

For indirect immunofluorescence, fixed cells were incubated for $1 \mathrm{~h}$ in blocking buffer (4\% BSA, 4 $\%$ goat serum $0,1 \%$ Triton), then overnight at $4^{\circ} \mathrm{C}$ with either anti-CDK5 (DC17, sc-249; Santa Cruz) or P35/p25 (Cell Signaling C64B10) diluted 1/500 in blocking buffer. Cells were then washed three times with PBS for $15 \mathrm{~min}$ and incubated for $1 \mathrm{~h}$ at room temperature with either Alexa488-conjugated anti-mouse or Alexa594-conjugated anti-rabbit antibodies (diluted 1/500), again followed by three washes in PBS for 15 min. Finally nuclei were stained with Hoechst 33342 (Sigma) diluted in $\mathrm{H}_{2} \mathrm{O}$ ( $1 \mu \mathrm{g} / \mathrm{mL}$ ) for $5 \mathrm{~min}$, and coverslips were finally washed in water and mounted on glass slides in Mowiol. Cells were imaged with a Zeiss Axioimager Z2 microscope equipped with a CoolSnap HQ2 camera and piloted by MetaMorph software. Excitation band / dichroic / emission band filters for imaging fluorescent signals were as follows : Hoechst : 340-380/400/450-490 nm ; Pep-CDKACT5Cy5 and Pep1-Cy5 were imaged using the Cy5 filters: 590-650/ 660/662-737 nm ; Alexa594conjugated antibodies were imaged using the Rhodamine filters; Alexa488-conjugated antibodies were imaged using the YFP filters : 450-490/495</500-550nm.

Confocal images were acquired with a Leica SP5-SMD inverted microscope equipped with an Argon LASER module and a $405 \mathrm{~nm}$ diode laser and 63X HCX Plan Apo CS oil 1.4NA objective. Each scan was acquired sequentially using constant laser intensity (15\% for $405 \mathrm{~nm}$ diode laser and $2 \%$ for Argon laser) with a $1200 \times 1200$ pixel resolution. Images acquired with Leica LAS-AF software were saved in .lif format and processed using Image $\mathrm{J}$ software.

For time-lapse live-cell imaging experiments, U87 cells were grown in a fluorodish (FD35-WPI) placed in an incubation chamber containing $5 \% \mathrm{CO}_{2}$ and thermostated at $37^{\circ} \mathrm{C}$. Confocal microscopy images were acquired automatically with a Zeiss Axioobserver/LSM 5 LIVE DUO inverted microscope equipped with a laser 510 LASER Module and 40X 1.3 EC plan NeoFluor Oil objective. In each dish, different fields were imaged sequentially using the Cy5, Cy3 or GFP filter every 5 min for $3 \mathrm{~h}$. Each scan was acquired at constant laser intensity (3\%) during $100 \mathrm{~ms}$ with a $512 \times 512$ pixel 
resolution. Images, acquired with ZEN software (Zeiss), were saved in .czi format and processed using Image $\mathbf{J}$ software. Live-cell imaging acquisitions of fluorescent cells were subtracted for background signal (minimal fluorescence) from a region that did not contain any cells. MetaMorph was used to build stacks of images (phase and fluorescence). For quantification of average PepCDKACT5-Cy5 and Pep-CDKACT5mut-Cy3 fluorescence at different time-points, a region of interest (ROI) delimiting 5-6 cells at each time point of the phase stacks was transferred to Cy5 and Cy3 stacks of the same time point (after background subtraction). Relative changes in CDK5 activity in living cells correspond to changes in PepCDKACT5-Cy5 fluorescence relative to PepCDKACT5mut-Cy3 over time.

\subsection{Statistical analyses.}

All data are summarized and presented as the mean \pm SEM from at least two or three representative and separate experiments or more whenever indicated. Statistical analyses were performed using a paired Student's t-test, with XLSTAT software (Addinsoft, Paris, France). Statistical significance was defined as: $* \mathrm{p} \leq 0.05, * * \mathrm{p} \leq 0.01$ and $* * * \mathrm{p} \leq 0.001$

\section{Results}

\subsection{Design and Characterization of a CDK5-specific Peptide Biosensor}

Although CDK5/p25 kinase constitutes an established biomarker, hyperactivity of which has been associated with neuronal pathologies, there are currently no tools available to probe its activity in a specific, sensitive and quantitative fashion. To address the lack of technologies available to quantify CDK5 activity, we designed and engineered a fluorescent peptide biosensor based on the CDKACT technology we previously developed [26, 32]. These biosensors are synthetic modular polypeptides which combine a kinase-specific substrate moiety onto which an environmentally-sensitive dye is conjugated, with a phosphoamino acid binding domain (PAABD), which specifically recognizes the substrate when it is phosphorylated by the kinase of interest. Kinase activity and biosensor phosphorylation are monitored through changes in fluorescence over time. Indeed, this favours PAABD binding to the substrate moiety, thereby affecting the local environment of the conjugated dye. In order to develop a CDK5-specific biosensor, and since CDK5 phosphorylates a wide variety of cytoskeletal proteins and neurofilaments, we engineered a library of bimodular peptide biosensors derived from distinct CDK5 substrates reported in the literature (Fig. 1A, Table 1) combined with an artificial PAABD derived from the WW domain of Pin1 [26, 33-38]. In the design of the biosensor peptide library, a unique cysteine residue was introduced within the substrate sequence, two residues 
upstream from the S/T-P phosphorylation site, so as to enable site-specific conjugation of a fluorescent dye proximal to the phosphorylation and PAABD interaction site. In order to verify the ability of these peptides to behave as reporters of CDK5 activity, they were first labelled with Cy5 and incubated with recombinant $\mathrm{CDK} 5 / \mathrm{p} 25$ in the presence of $\mathrm{ATP} / \mathrm{MgCl} 2$, in a thermostated fluorimeter. Fluorescence emission of $\mathrm{Cy} 5$ for each of these peptides was measured over time, revealing a significant enhancement of fluorescence emission intensity over time (Fig. 1B). In contrast, in the absence of kinase, these fluorescent peptides underwent progressive quenching in solution over time (Supporting Information Fig. S1), indicating that fluorescence enhancement was indeed due to the presence of the kinase.

CDK5 was initially identified through structural homology with CDK2 and both kinases phosphorylate several substrates in common, such as Histone H1 and Tau [7, 39]. Therefore, to assess the specificity of the different peptide biosensors, we asked whether they might respond preferentially to $\mathrm{CDK} 5$, by comparing their response to recombinant $\mathrm{CDK} 2 / \mathrm{Cyclin} \mathrm{A} 3$ or $\mathrm{CDK} 5 / \mathrm{p} 25$ in fluorescence-based kinase assays. Unexpectedly only one peptide biosensor, CDKACT5-ER104, derived from estrogen receptor (phosphorylation site 104) displayed greater selectivity for CDK5 over CDK2 (Fig. 1C, 1D and Supporting information Fig. S1). Further characterization of the CDKACT5-ER104 biosensor revealed that its response to CDK5 activity was dependent on the presence of $\mathrm{ATP} / \mathrm{MgCl} 2$, as well as on the presence of its p25 protein partner, since fluorescence emission was significantly lower in the absence of either of these (Fig. 1E). These results indicate that the biosensor effectively responds to CDK5 activity, residual fluorescence (30\%) being associated with CDK5 binding to the peptide biosensor. Moreover, they infer that p25 induces a conformational reorganization of CDK5 and consequently its enzymatic activity, as described for other CDK/Cyclin complexes, in particular CDK2/Cyclin A, in which Cyclin A converts CDK2 into a catalytically active conformation [40]. Finally, addition of ATP-competitive inhibitors Roscovitine, Dinaciclib or AT7519 led to a dose-dependent decrease in CDK5/p25-induced fluorescence enhancement of Cy5-CDKACT-ER104, to the same extent as removal of ATP/MgCl2, but had no significant effect on the basal fluorescence of the biosensor alone (Fig. 1F, Supporting information

Fig. S2 and S3). Taken together these results indicate that the Cy5-labelled CDKACT5-ER104 peptide biosensor responds to the catalytic activity of recombinant CDK5/p25 kinase complex in a dose-dependent fashion.

\subsection{Application of CDKACT5 to probe CDK5 activity in glioblastoma cell extracts}

To further validate the utility of CDKACT5-ER104 to probe CDK5 kinase activity in a more complex and physiologically-relevant environment, it was incubated with total protein lysates prepared from 
U87 glioblastoma cells, as a source of active CDK5/p25. As observed with the with recombinant CDK5/p25 kinase, glioblastoma cell extracts induced a significant and dose-dependent enhancement of Cy5-CDKACT-ER104 fluorescence (Fig. 2A, Supporting information Fig. S4 and S5), with an optimal response achieved with 150-200nM biosensor. Furthermore, this response was dependent on a dye which was sufficiently sensitive to its environment (Cy5) since conjugation of a non-responsive dye (Dylight 650 instead of Cy5) to the substrate domain did not yield any response. Biosensor response was equally dependent on the PAABD moiety, since incubation of Cy5-labelled ER104 substrate alone (no PAABD) did not yield any fluorescence enhancement in response to CDK5 activity in U87 cell extracts either. Likewise, an unrelated Cy5-labelled peptide (CDKACT Ctrl), bearing the same PAABD as CDKACT5 and a peptide sequence derived from ubiquitin, (which is not recognized by CDK5) were completely unresponsive (Fig. 2A, Supporting information Fig. S5). These results underscore the importance of the bipartite design comprising both a substrate and a PAABD in order to engineer a biosensor which responds to kinase activity, as well as the importance in the choice of the fluorescent dye conjugated to the substrate moiety.

To further address the specificity of biosensor response to endogenous CDK5, it was incubated with CDK5 inhibitors Roscovitine and Dinaciclib, which indeed inhibited the fluorescent enhancement of Cy5-CDKACT-ER104 induced upon incubation with U87 cell extracts in a dose-dependent fashion. In contrast, neither the CDK inhibitors PD-0332991 (CDK4/6 inhibitor) and RO3306 (CDK1 inhibitor), nor the MEK1 and MEK2 - MAPK inhibitor U0126, or the Src, Lck, Fyn and Hck inhibitor PP2 had any significant effect on the response of the biosensor to CDK5 activity in U87 cell extracts (Fig. 2B and 2C, Supporting information Fig. S6 and S7).

Conversely we asked whether CDKACT5-ER104 peptide biosensor could report on CDK5 activation in cells treated with ionomycin, which promotes intracellular calcium influx, with retinoic acid, which induces neuronal differentiation, or following starvation by serum deprivation, which induces cellular stress and consequently calcium influx. Indeed CDK5 activation by each of these treatments could be monitored through sensitive (and transient) changes in CDKACT5-Cy5 fluorescence (up to 20\% relative to untreated cells) (Fig. 2D).

Finally, CDKACT5-ER104-Cy5 was used to evaluate the specific activity of CDK5 kinase in cell extracts from a panel of neuronal or non-neuronal cell lines (Fig. 2E). Specific CDK5 activity was determined as the relative kinase activity measured with CDKACT5-ER104-Cy5 biosensor with respect to the relative CDK5 protein levels quantified by Western blotting in $10 \mu \mathrm{g}$ of total protein from normalized cell lysates (Supporting Information Fig. S8). Whilst Western blot analysis revealed high levels of CDK5 kinase in HeLa, MDA, A375, PC12, Gli4 and Gli7, biosensor profiling 
and determination of specific kinase activity revealed it was much lower in PC12 cells than in U87, C6 and SNB75 glioblastoma cell lines. Gli4 and Gli7 cell lines derived from patients also displayed low CDK5 activity relative to CDK5 protein levels. HeLa, MDA-MB-231 and A375 exhibited similar specific CDK5 activity to U87 cells, in agreement with reports in the literature inferring that CDK5 activity is deregulated in these cell lines.

Taken together these results reveal that the CDKACT5 peptide biosensor, comprising a short artificial phosphorecognition domain derived from the WW domain of Pin1 and a substrate sequence derived from ER, constitutes a specific and sensitive tool to assess the relative activity of CDK5 using cell extracts as a source of kinase, and following treatment with selective inhibitors or activating stimuli.

\subsection{Quantification of CDK5 activity in U87 cells by fluorescence imaging}

Having established that CDKACT5 could respond to CDK5 kinase in a specific and sensitive fashion in U87 cell extracts, we further asked whether it could be implemented to monitor CDK5 activity directly within living cells, and used to quantify differences in CDK5 activity upon treatment with drugs. To this aim, we designed a cell-penetrating variant of the biosensor labelled with Cy5 (PepCDKACT5-Cy5), which was efficiently internalized into cultured U87 cells (Fig. 3A, 3B, 3C and and Supporting Information Fig. S9). This biosensor colocalized with both endogenous CDK5 and p25 in this glioblastoma cell line, inferring it might recognize endogenous CDK5 in living cells (Supporting information Fig. S10). In order to assess the ability of Pep-CDKACT5-Cy5 to report on CDK5 activity, it was internalized into U87 cells which had been pretreated or not with $10 \mu \mathrm{M}$ CDK5 inhibitors Roscovitine and Dinaciblib for $24 \mathrm{~h}$. Following biosensor internalization, cells were fixed and nuclei were stained with Hoechst, then observed by fluorescence microscopy to determine the ratio of $\mathrm{Cy} 5 / \mathrm{Hoech}$ st fluorescence. Quantification of $\mathrm{Cy} 5 /$ Hoechst fluorescence in several regions of interest comprising 3-4 cells each revealed an average 60\% decrease in biosensor fluorescence associated with Dinaciclib treatment, and a corresponding 80\% reduction following Roscovitine treatment. These ratiometric quantifications revealed that cells treated with inhibitors had significantly lower Cy5/Hoechst fluorescence ratio, associated with lower CDKACT5-Cy5 fluorescence emission as a consequence of CDK5 inhibition, in agreement with results obtained in vitro in assays performed with recombinant protein or cell extracts. In contrast, Cy5/Hoechst fluorescence in U87 cells internalized with Cy5-labelled Pep1 was essentially unaffected by Roscovitine or Dinaciclib treatment (Fig. 3D and 3E and Supporting Information Fig. S11).

\subsection{Imaging the dynamics of CDK5 activation in U87 cells in real-time by timelapse microscopy}


CDK5 activation is believed to occur first upon cleavage of p35 to p25 by calpains at the cell membrane, thereby enabling its diffusion into the cytoplasm where it can encounter and phosphorylate its substrates (Fig. 4A). In order to visualize the dynamics of CDK5 activation in realtime and ask whether its activation was associated with intracellular calcium influx, we decided to implement the CDKACT biosensor to monitor CDK5 activity in U87 cells by time-lapse microscopy. To this aim, we either induced calcium influx artificially by treating cells with ionomycin, or prompted a more physiological calcium influx, by flushing cells with $\mathrm{KCl}$ to change their membrane potential. In a first set of experiments, U87 cells were overlaid with the PepCDKACT5-Cy5 for one hour and imaged to achieve a stable signal, and then treated (or not) with $10 \mu \mathrm{M}$ ionomycin whilst performing time-lapse imaging. Images were acquired every $5 \mathrm{~min}$ and average fluorescence intensity at the cell membrane or in the cytoplasm was plotted against time (Fig. 4B and C). This first set of experiments revealed that CDK5 activation peaks at the cell membrane after 100 minutes treatment with ionomycin, closely followed by its activation in the cytoplasm, in line with the model presented in Fig. 4A, in which CDK5 activation is believed to occur upon cleavage of p35 to p25, thereby enabling its diffusion into the cytoplasm where it can encounter and phosphorylate its substrates. In contrast a mutant biosensor, Pep-CDKACT5mut-Cy5, in which the phosphorylation site within the substrate sequence is replaced by a non-phosphorylatable alanine, did not yield any significant changes in fluorescence intensity over time and were not observed accumulating at the cell membrane at any time (Supporting information Fig. S12).

In a second set of experiments, U87 cells were grown in a dish which was flushed with $100 \mathrm{mM} \mathrm{KCl}$ to stimulate physiological changes in membrane potential that promote calcium influx. These cells were overlaid with PepCDKACT5-Cy5, as well as Fluo-4 AM sensor used to monitor calcium influx over time. Acquisition of fluorescence signals over time indeed revealed that PepCDKACT5 responded to changes in CDK5 activity in living cells, which were intimately associated with intracellular oscillations in calcium levels time (Fig. 4D and E).

Taken together, these imaging studies reveal that a self-cell-penetrating derivative of CDKACT5ER104 biosensor is a reliable and sensitive reporter of CDK5 activity that can report on CDK5 activity and inhibition directly within living cells. Moreover we have shown that this biosensor can be used to investigate the dynamics of CDK5 activation in space and in time. Hence our studies provide new evidence which completes the static model from the literature describing an inactive form of CDK5 anchored at the membrane through association with p35, versus an active form complexed to p25 in the cytoplasm following calcium-induced calpain-mediated proteolysis. The CDKACT5-ER104 biosensor indeed reveals initial activation of CDK5 at the membrane, which then progressively 
diffuses into the cytoplasm. Moreover time-lapse imaging of intracellular physiological calcium pulses revealed that these are indeed intimately associated with CDK5 activation.

\section{Discussion}

In this study we describe the design and characterization of an original fluorescent peptide biosensor which reports on the activity of CDK5 kinase in a sensitive and selective fashion. This chemical reporter constitutes a versatile tool which provides means of quantifying the relative activity of CDK5 both in vitro, in complex biological samples such as cell extracts, as well as in living cells by fluorescence microscopy, to image the dynamics of kinase activation in space and in time.

Specificity, selectivity and sensitivity are essential features in the process of biosensor design and validation. In the case of fluorescent peptide biosensors this implies that three criteria be verified and met: target recognition, probe sensitivity and signal transduction. First, to optimize the specificity of kinase recognition, and since CDK5 was initially discovered through homology with CDK2, and phosphorylates several substrates in common such as Tau and Histone [7, 39], we sought to identify preferential response to $\mathrm{CDK} 5 / \mathrm{p} 25$ over CDK2/cyclin A activity using a small library of peptide biosensors derived from substrates of CDK5 [33-38]. This approach proved extremely useful since it allowed us to identify a single peptide biosensor displaying selectivity for CDK5 over CDK2, derived from the ER receptor (phosphorylation site 104). Further characterization of this biosensor revealed that it naturally detected CDK5 (due to recognition of the substrate moiety by the kinase) but that maximal fluorescence enhancement was only achieved in response to catalytic activity of CDK5, which is dependent on $\mathrm{ATP} / \mathrm{MgCl} 2$, and counteracted by incubation with Roscovitine, Dinaciclib and AT7519. In a set of complementary experiments using cell extracts as a source of kinase and inhibitors, we observed a significant reduction in CDKACT5-ER104-Cy5 response upon addition of Roscovitine and Dinaciclib, whereas CDK1 and CDK4/6 inhibitors, as well as U0126 and PP2 inhibitors had no significant effect, supporting selective response of the biosensor to CDK5 over CDK1, CDK2, CDK4/6, MEK1/MEK2 MAPK and Src/Lyn/Hck. In particular, the selectivity of CDKACT5-ER104-Cy5 for CDK5 over CDK4 makes it a very attractive tool for investigating signaling pathways in glioblastoma, in combination with our previously developed CDK4-selective biosensor [26], to assess whether specific glioblastoma cell lines or tumour biopsies are associated with CDK5 hyperactivation, and/or with CDK4 hyperactivation. Since both kinases are potentially upregulated in glioblastoma [44], biosensor quantification of relative kinase activities could guide medical treatment. 
Moreover we have shown that the CDKACT-ER104-Cy5 could respond to CDK5 stimulation by retinoic acid, ionomycin treatment or starvation of U87 cells, through a sensitive increase in fluorescence enhancement relative to untreated cells. Hence this peptide biosensor constitutes an attractive tool to evaluate both CDK5 stimulation and inhibition in sensitive and dose-dependent fashion, which can be implemented to evaluate the efficacy of new inhibitors targeting CDK5 in a physiologically-relevant environment, in the context of drug discovery programmes.

The biosensor described in this study relies on a bimodular system, in which a phospho-amino acid binding domain (PAABD) linked to a kinase-specific substrate moiety preferentially interacts with the latter when it is phosphorylated by an active kinase. This alters the local environment of a fluorescent dye conjugated proximal to the phosphorylation site and consequently affects its fluorescence emission properties. We have shown that the choice of the dye conjugated onto the substrate moiety is detrimental for biosensor response and that Cy5 was a good choice for our CDK5 activity reporter. Its replacement by Dylight 650 completely abolished fluorescence enhancement associated with CDK5 activity. We also demonstrated the importance of the PAABD in this bipartite construct, since the peptide substrate moiety alone conjugated to $\mathrm{Cy} 5$ did not respond to CDK5 activity at all.

In this study we have further used CDKACT5-ER104 biosensor to determine the specific kinase activity of CDK5 in a panel of neuronal and non-neuronal cell lines. We have shown that high CDK5 and p35/p25 protein expression levels did not necessarily correlate with high kinase activity. Such profiling studies infer that CDK5 activity is regulated at the posttranslational level, through phosphorylation and/or interactions with regulatory partners. Although in vitro studies by Peterson et al. showed that CDK5 activity is essentially identical when associated to p25 and p35 [39], the work of Rosales reveals that CDK5 is activated by phosphorylation on S159 in mouse brain extracts [42]. CDK5 has also more recently been found to associate with Cyclin E, which may play a role in the overall downregulation of kinase activity [43]. We have previously reported similar findings with a biosensor of CDK4 activity, which reports on the differential activity of this kinase in melanoma cell lines harbouring different genetic mutations in the p16/CDK4/CyclinD/Rb pathway [32]. Indeed, protein kinases are more often than not regulated at the posttranslational level, through phosphorylation, as well as through interaction with activating partners and endogenous inhibitors that modulate their catalytic activity, their conformation and/or their subcellular localization.

Last but not least, we have shown that a self-cell-penetrating variant of CDKACT5 biosensor enabled quantification of CDK5 activity in living cells by fluorescence imaging and ratiometric quantification of biosensor fluorescence relative to that of Hoechst. We further implemented this cell-penetrating 
biosensor to image the spatio-temporal dynamics of CDK5 activation, thereby gaining insight into the kinetics of CDK5 activation associated with changes in its subcellular localization and demonstrating its intimate correlation with calcium influxes by time-lapse imaging. Our results complete the static model describing inactive CDK5 anchored at the membrane when associated with p35, versus an active form of CDK5/p25 released into the cytoplasm following calcium-induced calpain-mediated cleavage of p35 to p25. The CDKACT5-ER104 biosensor indeed reveals that initial activation of CDK5 occurs at the cell membrane, in agreement with recent reports describing phosphorylation of membrane - bound receptors by CDK5 [41]. CDK5 activity then most likely diffuses progressively into the cytoplasm where it encounters the larger number of cytoskeletal substrates.

Biosensor technologies that report on enzyme function constitute important tools that provide a reflection of biomolecular behaviour which accounts for the influence of regulatory signals on overall kinase activity, in contrast with genetic, transcriptomic or proteomic approaches which inform on gene, transcript or protein expression levels. There are however very few kinase activity reporters which enable sensitive and selective quantification of kinase activity in vitro, in extracto and in living cells. Among the renowned commercially available systems used to evaluate kinase activities in vitro, fluorescently-labelled ATP and/or phospho-specific antibody-based systems such as the Universal Protein Assay $\mathrm{Kit}^{\mathrm{TM}}$, ADAPTA ${ }^{\mathrm{TM}}$, KINATIV ${ }^{\mathrm{TM}}$, LANTHASCREEN $^{\mathrm{TM}}$, or the more recent peptidebased PHOSPHOSENS ${ }^{\mathrm{TM}}$ biosensors do not provide the versatility and selectivity of our system.

CDKACT5 biosensor constitutes a versatile tool which can be applied to monitor CDK5 activity in vitro by fluorescence spectroscopy, using cell extracts as a source of kinase activity, as well as in living cells by time-lapse fluorescence microscopy. By affording means of monitoring alterations in CDK5 activity associated with disease, this fluorescent biosensor constitutes a first-of-a kind chemical tool to evaluate the functional status of a well established biomarker in glioblastoma, neuroblastoma and several neurodegenerative pathologies. We therefore anticipate it could enable development of a predictive diagnostic assay based on detection of an enzymatic activity, thereby complementing current antigenic and genetic diagnostic approaches, and could further contribute to patient stratification. Since CDKACT5 responds to CDK5 inhibition, it could further serve to develop point-of-care companion assays by monitoring the specific benefit of targeted therapeutic intervention.

\section{ACKNOWLEDGEMENTS}

This work was supported by the CNRS (Centre National de la Recherche Scientifique) and a grant from the GSO Canceropole to MCM. MP was supported by a fellowship from the CBS2 doctoral 
school of Montpellier University. We acknowledge the MRI imaging facility (Montpellier, France), a member of the national infrastructure France-BioImaging.

\section{References}

[1] Stupp R. et al. Effects of radiotherapy with concomitant and adjuvant temozolomide versus radiotherapy alone on survival in glioblastoma in a randomised phase III study: 5-year analysis of the EORTC-NCIC trial. Lancet Oncology, 2009, 10, 459-466

[2] Dhavan, R., Tsai, L.H. A Decade of CDK5. Nature Reviews. Molecular Cell Biology 2001, 2, $749-59$.

[3] Smith D.S., Greer P.L.,Tsai LH. Cdk5 on the brain. Cell Growth \& Differentiation, 2001, 12, $277-$ 283

[4] Smith DS, Tsai LH. Cdk5 behind the wheel: a role in trafficking and transport? Trends Cell Biol. $2002,12,28-36$.

[5] Smith D. Cdk5 in neuroskeletal dynamics. Neurosignals. 2003, 12, 239-51.

[6] Cheng K, Ip NY. Cdk5: a new player at synapses. Neurosignals. 2003, 12, 180-90.

[7] Hellmich M.R., Pant H.C., Wada E., Battey J.F. Neuronal cdc2-like kinase: a cdc2-related protein kinase with predominantly neuronal expression. Proc. Natl. Acad. Sci. USA. 1992, 89, 10867-71

[8] Tsai LH, Takahashi T, Caviness VS Jr, Harlow E. Activity and expression pattern of cyclindependent kinase 5 in the embryonic mouse nervous system. Development. 1993, 119, 1029-40.

[9] Ino H, Ishizuka T, Chiba T, Tatibana M. Expression of CDK5 (PSSALRE kinase), a neural cdc2related protein kinase, in the mature and developing mouse central and peripheral nervous systems. Brain Res. 1994, 661, 196-206

[10] Lew J. et al. A brain-specific activator of cyclin-dependent kinase 5. Nature, 1994, 371, 423-426

[11] Su SC, Tsai LH. Cyclin-dependent kinases in brain development and disease. Annu Rev Cell Dev Biol. 2011, 27, 465-91.

[12] Nikolic M, Dudek H, Kwon YT, Ramos YF, Tsai LH. The cdk5/p35 kinase is essential for neurite outgrowth during neuronal differentiation. Genes Dev. 1996, 10, 816-25.

[13] Gupta A, Tsai LH. Cyclin-dependent kinase 5 and neuronal migration in the neocortex. Neurosignals. 2003, 12, 173-9. 
[14] Cruz, J.C.,Tsai, L.H. A Jekyll and Hyde kinase: roles for Cdk5 in brain development and disease. Curr. Opin. Neurobiology, 2004, 14, 390-94

[15] Crews, L., Masliah, E., Molecular mechanisms of neurodegeneration in Alzheimer's disease. Hum. Mol. Genet. 2010, 19, R12-20

[16] Cheung, Z.H.,Ip, N. Y. Cdk5: a multifaceted kinase in neurodegenerative diseases. Trends Cell Biol. 2012, 22, 169-175.

[17] Kimura T, Ishiguro K, Hisanaga S. Physiological and pathological phosphorylation of tau by Cdk5. Front Mol Neurosci. 2014, 7, 65

[18] Catania A et al. Expression and localization of cyclin-dependent kinase 5 in apoptotic human glioma cells. Neuro Oncol. 2001, 3, 89-98.

[19] Yushan, R., et al. Insights into the clinical value of cyclin-dependent kinase 5 in glioma: a retrospective study. World J Surg Oncol 2015, 13, 223.

[20] Liu, R., Tian, B., Gearing, M., Hunter, S., Ye, K., Mao, Z. Cdk5-mediated regulation of the PIKE-A-Akt pathway and glioblastoma cell invasion. Proc. Natl. Acad. Sci. U.S.A. 2008, 105, 75707575.

[21] Xie, Q., et al. Mitochondrial control by DRP1 in brain tumor initiating cells. Nat Neurosci. 2015, $18,501-10$

[22] Tsai L.H. et al. p35 is a neural-specific regulatory subunit of cyclin-dependent kinase 5. Nature, 1994, 371, 419-423

[23] Asada A, Yamamoto N, Gohda M, Saito T, Hayashi N, Hisanaga S. (2008) Myristoylation of p39 and p35 is a determinant of cytoplasmic or nuclear localization of active cyclin-dependent kinase 5 complexes. J Neurochem. 2008, 106, 1325-36

[24] Hashiguchi M. et al. Truncation of CDK5 activator p35 induces intensive phosphorylation of Ser202/Thr205 of human Tau. J. Biol. Chem. 2002, 277, 44525-530

[25] Kusakawa, G.-I., Saito,T., Onuki,K.,Ishiguro, R., Kishimoto, T. Hisanaga, S.-I. Calpaindependent proteolytic cleavage of the p35 cyclin-dependent kinase 5 activator to p25. J Biol Chem. 2000, 275, 17166-17172

[26] Prével, C., et al. Fluorescent peptide biosensor for monitoring CDK4/Cyclin D hyperactivity in melanoma cells, mouse xenografts and skin biopsies. Biosensors and Bioelectronics, 2016, 85, 371380.

[27] Morris M.C. et al. A peptide carrier for the delivery of biologically active proteins into mammalian cells. Nat. Biotechnol. 2001, 19, 1173-6

[28] Kurzawa, L., Pellerano, M., Coppolani, JB., Morris, M.C. Fluorescent peptide biosensor for probing the relative abundance of cyclin-dependent kinases in living cells. PLoS One 2011, 6, e26555 
[29] Morris M.C., Gondeau C., Tainer JA, Divita G. Kinetic mechanism of activation of the Cdk2/cyclin A complex. Key role of the C-lobe of the Cdk. J Biol Chem. 2002, 277, 23847-53

[30] Gondeau C, Gerbal-Chaloin S, Bello P, Aldrian-Herrada G, Morris MC, Divita G. Design of a novel class of peptide inhibitors of cyclin-dependent kinase/cyclin activation. J Biol Chem.2005, 280, 13793-800.

[31] Corbel C, Zhang B, Le Parc A, Baratte B, Colas P, Couturier C, Kosik KS, Landrieu I, Le Tilly V, Bach S. Tamoxifen inhibits CDK5 kinase activity by interacting with p35/p25 and modulates the pattern of tau phosphorylation. Chemistry \& Biology 2015, 22, 472-482

[32] Van TNN, Pellerano M., Lykaso S., Morris M.C. Fluorescent protein biosensor for probing CDK/Cyclin activity in vitro and in living cells. ChemBioChem. 2014, 15, 2298-305

[33] Contreras-Vallejos et al. Searching for novel Cdk5 substrates in brain by comparative phosphoproteomics of wild type and Cdk5-/- mice. PlosOne 2014, 9, e90363.

[34] Wada Y. et al. (1998) Microtubule-stimulated phosphorylation of tau at Ser202 and Thr205 by cdk5 decreases its microtubule nucleation activity. J Biochem. 124, 738-46.

[35] Tian B., Yang Q. and Mao Z. (2009) Phosphorylation of ATM by Cdk5 mediates DNA damage signalling and regulates neuronal death. Nat. Cell. Biol. 11, 211-218

[36] Wen Z. et al. (2014) CDK5-mediated phosphorylation and autophagy of RKIP regulate neuronal death in Parkinson's disease. Neurobiology Aging, 1-11

[37] Bibb J.A. et al. (1999) Phosphorylation of DARPP-32 by Cdk5 modulates dopamine signalling in neurons. Nature, 402, 669-671

[38] Matsubara M., Kusubata M., Ishiguro K., Uchida T., Titani K. and Taniguchi H. (1996) Sitespecific phosphorylation of synapsin I by mitogen-activated protein kinase and Cdk5 and its effects on physiological functions. J.Biol.Chem. 271, 21108-13

[39] Peterson DW et al. (2010) No difference in kinetics of tau or histone phosphorylation by CDK5/p25 versus CDK5/p35 in vitro. Proc Natl Acad Sci U S A., 107, 2884-9.

[40] Jeffrey PD, Russo AA, Polyak K, Gibbs E, Hurwitz J, Massagué J, Pavletich NP. Mechanism of CDK activation revealed by the structure of a cyclinA-CDK2 complex. Nature, 1995, 376, 313-20.

[41] Duhr D., et al. (2014) Cdk5 induces constitutive activation of 5-HT6 receptors to promote neurite growth. Nat. Chem. Biol. 10, 590-97

[42] Rosales J.L., Han B., and Lee K.L. (2003) Cdk7 functions as a Cdk5 activating Kinase in brain. Cell. Physiol. Biochem. 13, 285-296.

[43] Odajima J. et al. (2011) Cyclin E constrains Cdk5 activity to regulate synaptic plasticity and memory formation. Dev Cell. 21, 655-68.

[44] Brennan C.W. et al. (2013) The somatic genomic landscape of glioblastoma. Cell. 155, 462-77. 
Fluorescent reporter of CDK5 activity

TABLE 1

\begin{tabular}{|l|l|l|c|}
\hline CDKACT5 Biosensor & substrate sequence & Substrate & $\begin{array}{c}\text { Phosphorylation } \\
\text { site }\end{array}$ \\
\hline Biosensor A (Tau205) & SGYSSPGSCSTPGSR & TAU & T205 \\
\hline Biosensor B (Tau404) & KSPVVSGDCSSPR & TAU & S404 \\
\hline Biosensor C (ATM) & TKKCSS PNKIAS & ATM & S794 \\
\hline Biosensor D (R-KIP) & LGKCSTPTQVK & R-KIP & T42 \\
\hline Biosensor E (DARPP) & NPCSYTPPSLKAVQRIA & DARPP & T75 \\
\hline Biosensor F (SYN) & RPPACSSPQRQA & Synuclein & S553 \\
\hline Biosensor G (ER104) & LNSVCSSPLML & ER1 & S104 \\
\hline Biosensor H (ER118) & PQCSSPFLQPH & ER1 & S118 \\
\hline
\end{tabular}




\section{FIGURE LEGENDS}

\section{FIGURE 1 - Design and characterization of CDKACT5 biosensors with recombinant kinase}

(A) Schematic representation of CDKACT5 peptide biosensors (B) Relative changes in fluorescence intensity over time of 200nM Cy5-labelled peptide biosensors incubated with $100 \mathrm{nM} \mathrm{CDK5/p25}$ or (C) 50nM CDK2/Cyclin A3. (D) Histogram representing the relative fluorescence enhancement of the different peptide biosensors in response to CDK5 or to CDK2 activity (E) Relative changes in fluorescence intensity of $200 \mathrm{nM}$ of Cy5-labelled CDKACT5-ER104 incubated with $100 \mathrm{nM}$ of $\mathrm{CDK} 5 / \mathrm{p} 25$ in presence of $\mathrm{ATP}, \mathrm{MgCl}_{2}$ or absence of $\mathrm{ATP} / \mathrm{MgCl}_{2}$, and $\mathrm{CDK} 5$ with $\mathrm{ATP} / \mathrm{MgCl}_{2}$. $(\mathbf{F})$ Relative changes in fluorescence intensity of $200 \mathrm{nM}$ of Cy5-labelled CDKACT5-ER104 incubated with $100 \mathrm{nM}$ of $\mathrm{CDK} 5 / \mathrm{p} 25+\mathrm{ATP} / \mathrm{MgCl} 2$ in the presence of $20 \mu \mathrm{M}$ Roscovitine, Dinaciclib or AT7519. All fluorescent activity assays ( $\lambda$ exc: $649 \mathrm{~nm} / \lambda \mathrm{em}: 670 \mathrm{~nm}$ ) were performed in PBS buffer.

A.

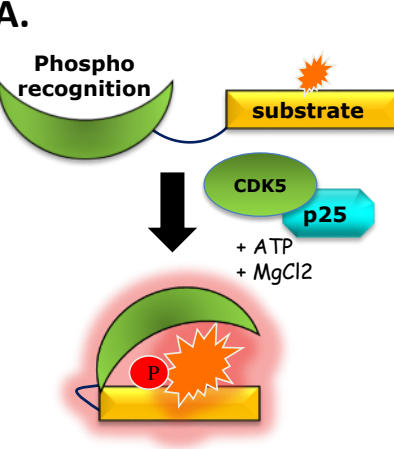

D.

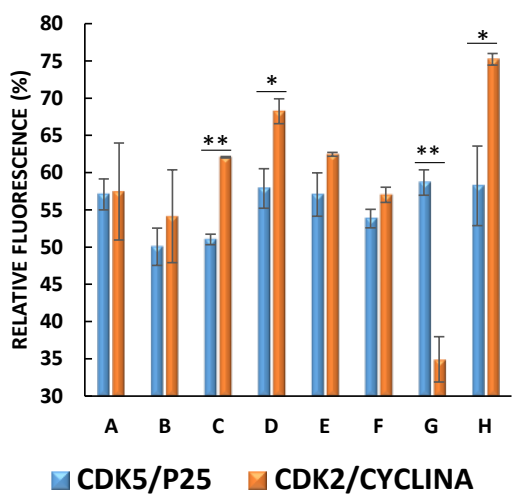

B.

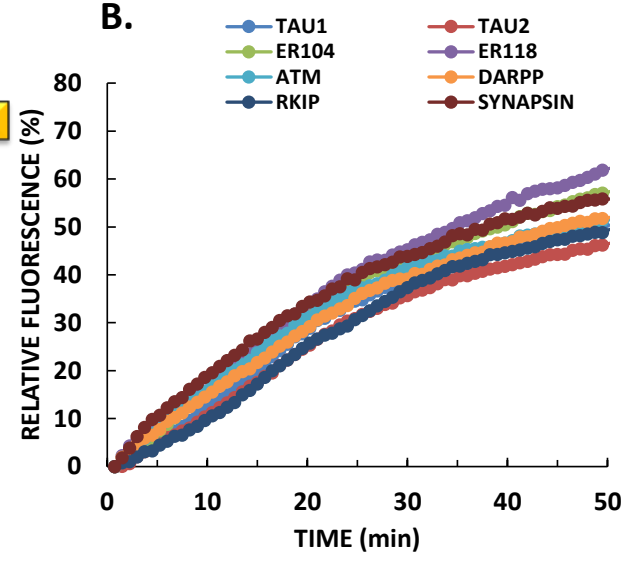

E.

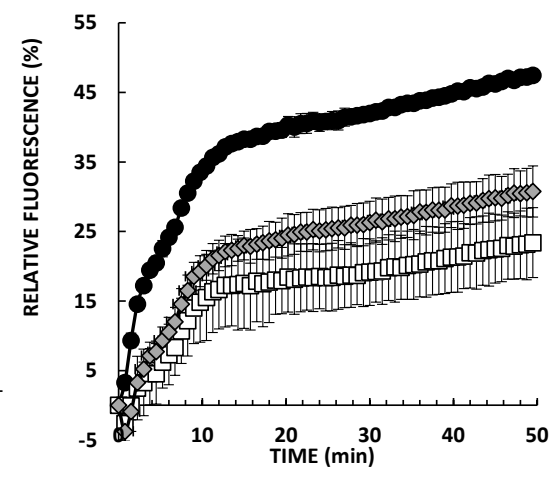

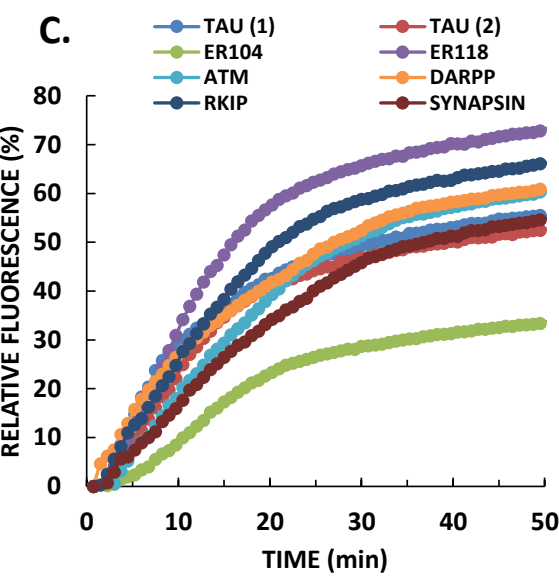

F.

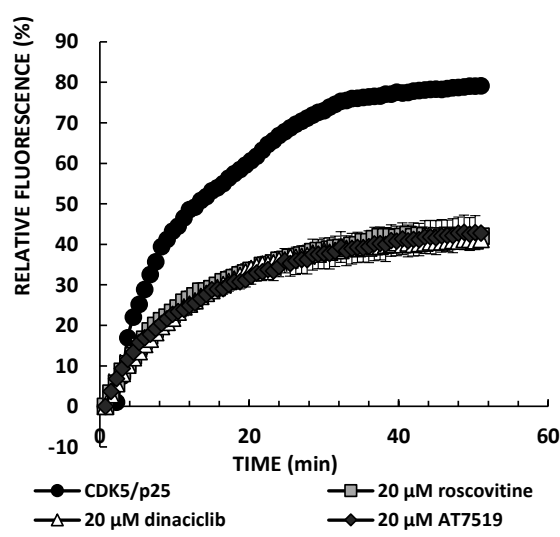




\section{FIGURE 2 -Application of CDKACT5 to probe/report on CDK5 activity in cell extracts}

(A) Relative changes in fluorescence of $200 \mathrm{nM}$ Cy5-labelled CDKACT5-ER104, of Dylight650labelled CDKACT5-ER104, of Cy5-labelled ER104 substrate and of Cy5-labelled CDKACT-Ctrl peptide were monitored over time (B) Relative fluorescence of $200 \mathrm{nM}$ Cy5-labelled CDKACT5ER104 upon incubation with $5 \mu \mathrm{g}$ U87 cell extracts and different concentrations of Roscovitine or (C) other kinase inhibitors (D) Stimulation of CDK5 activity was observed upon treatment with ionomycin for $1 \mathrm{~h} 30$ (stimulates entry of calcium into cells); retinoic acid (induces neuronal differentiation) and starvation by serum deprivation (E) Specific activity of CDK5 kinase in cell extracts from neuronal and non-neuronal cell lines (relative activity / protein levels) and Western blotting of CDK5 and p25/p35 protein levels in $10 \mu \mathrm{g}$ of total protein from normalized cell lysates.

A.

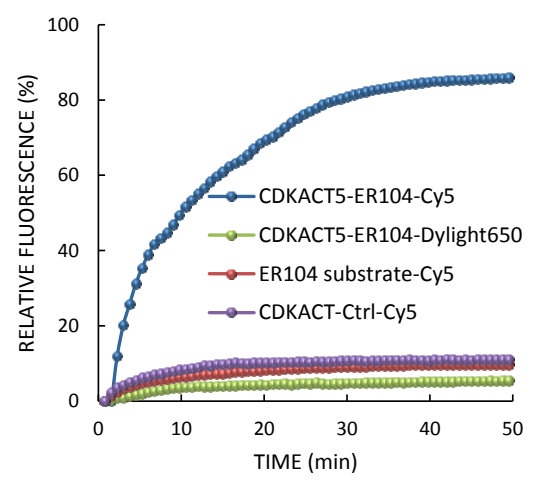

D.

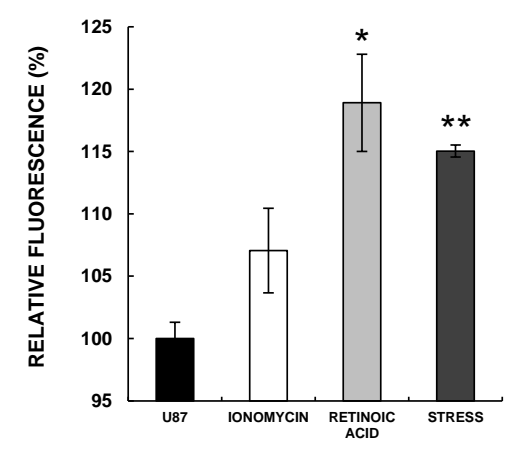

B.

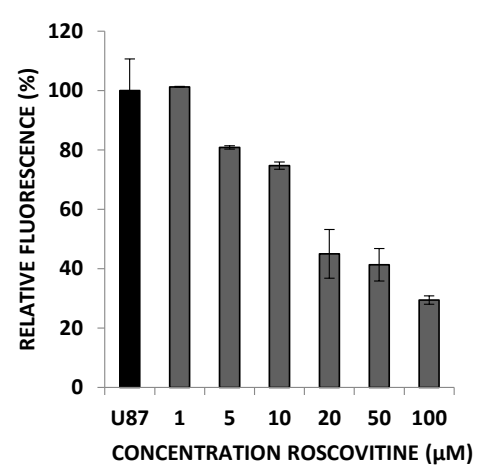

E.

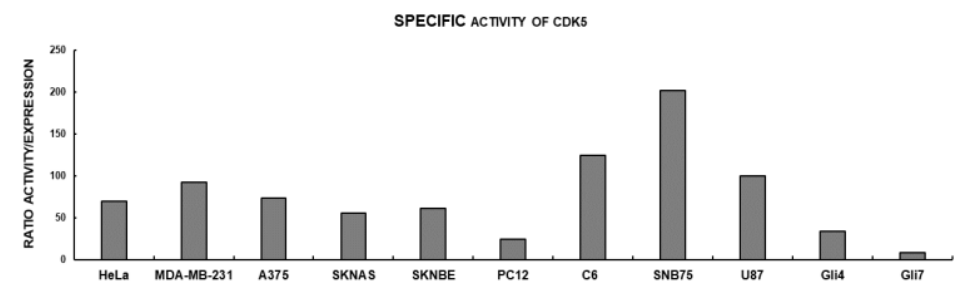




\section{FIGURE 3 - Quantification of CDK5 activity in U87 cells by fluorescence microscopy}

(A) Schematic representation of the cell-penetrating peptide biosensor Pep-CDKACT5-Cy5 and its translocation through the cell membrane (B) Fluorescence micrographs of cells overlaid with no peptide (mock), with CDKACT5-Cy5 peptide or with Pep-CDKACT5-Cy5. Left panels correspond to Hoechst staining of nuclei; right panels to fluorescence emission in the Cy5 filter (C) Representative confocal images of U87 cells showing Pep-CDKACT5-Cy5 has indeed penetrated into cells. (D) Relative ratiometric quantification of Cy5/Hoechst in cells internalized with PepCDKACT-Cy5 and treated with $10 \mu \mathrm{M}$ Dinaciclib or Roscovitine, compared to mock-treated cells.

(E) Relative ratiometric quantification of Cy5/Hoechst in cells internalized with Pep-1-Cy5 which were treated with $10 \mu \mathrm{M}$ Dinaciclib or Roscovitine. *** : p value $<0,001$

A.

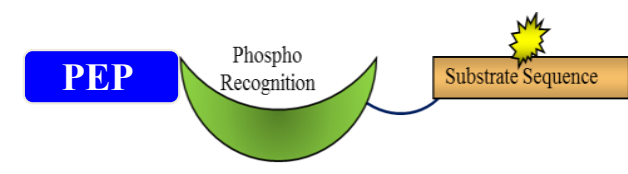

B.

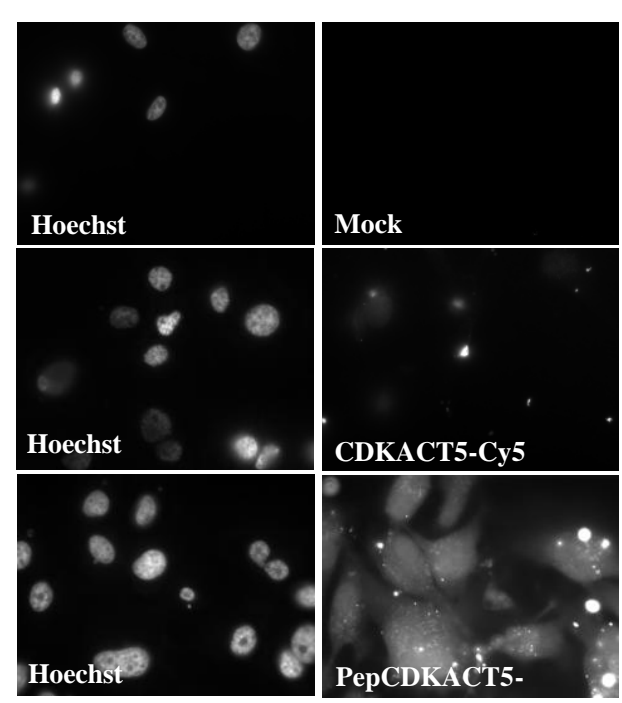

C.
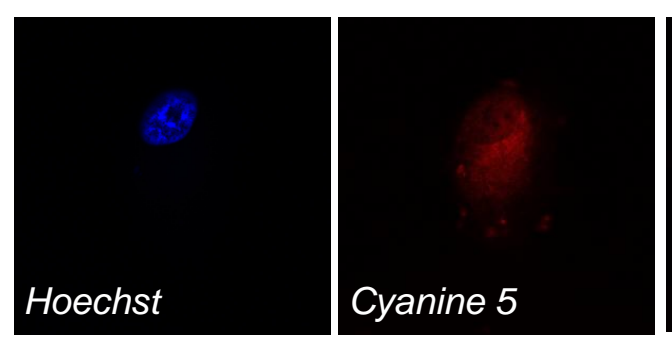
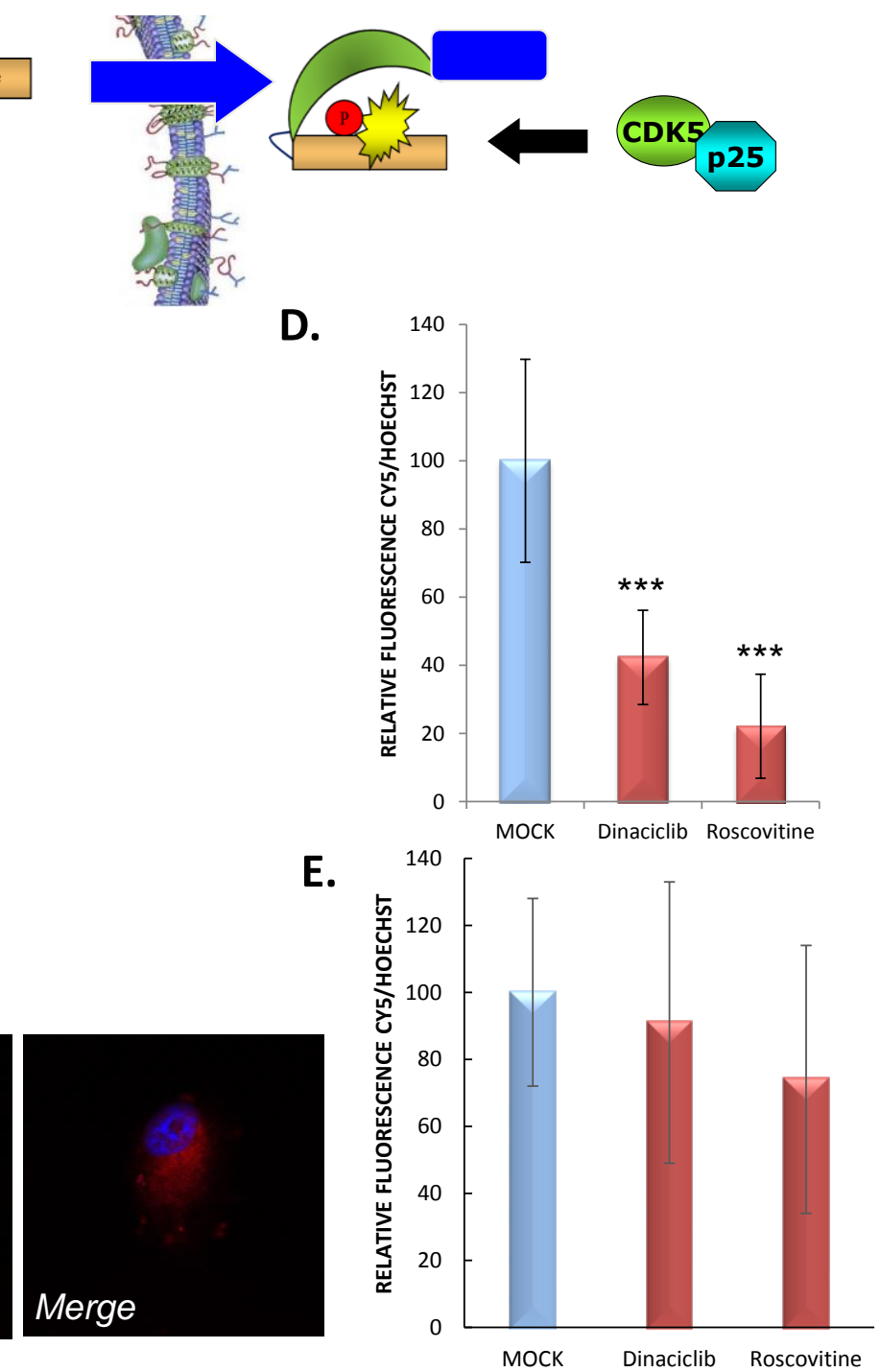
FIGURE 4 - Imaging the dynamics of CDK5 activation in U87 cells in real-time by timelapse microscopy

(A) Schematic representation of CDK5 activation at the cell membrane associated by Ca-dependent activation of calpains, which cleave p35 into p25, thereby releasing CDK5/p25 into the cytoplasm. (B) Representative micrographs of U87 cells overlaid with the PepCDKACT5-Cy5; upper panel after $20 \mathrm{~min}$ just prior to addition of ionoycin; lower panel after $100 \mathrm{~min}$, following addition of $10 \mu \mathrm{M}$ ionomycin. (C) Fluorescence intensity plotted against time at the cell membrane or in the cytoplasm. (D) Micrographs of U87 cells overlaid with the Pep-CDKACT5-ER104-Cy5 biosensor and Fluo-4 AM which reports on calcium presence/ influxes. (E) Oscillations in fluorescence intensity of PepCDKACT5-ER104-Cy5 biosensor and Fluo-4 AM were imaged and plotted over time in single U87 cells flushed with $100 \mathrm{mM} \mathrm{KCl}$.

A. Calcium influx

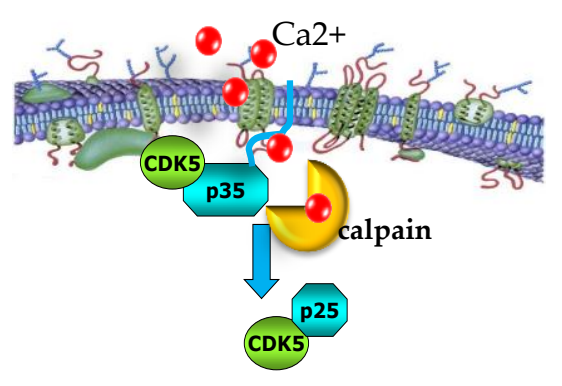

B.

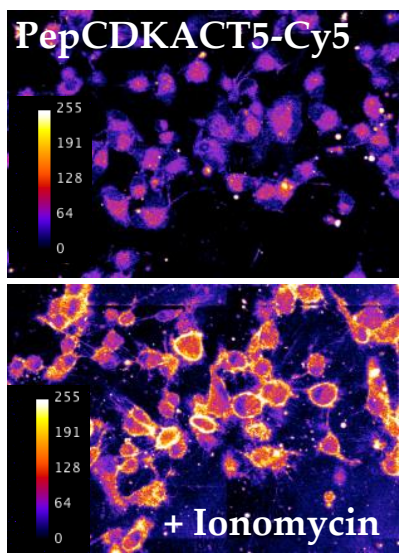

D.

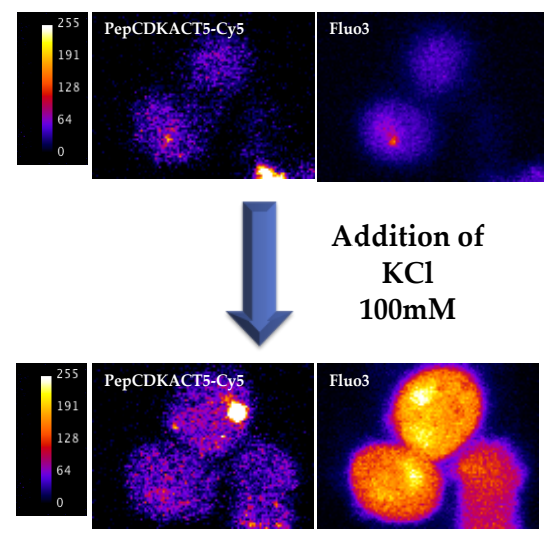

C.

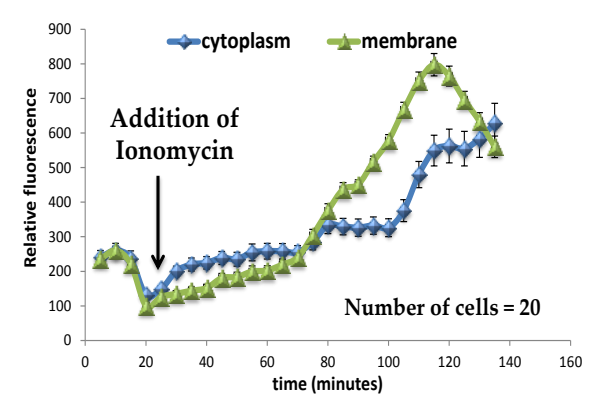

E.

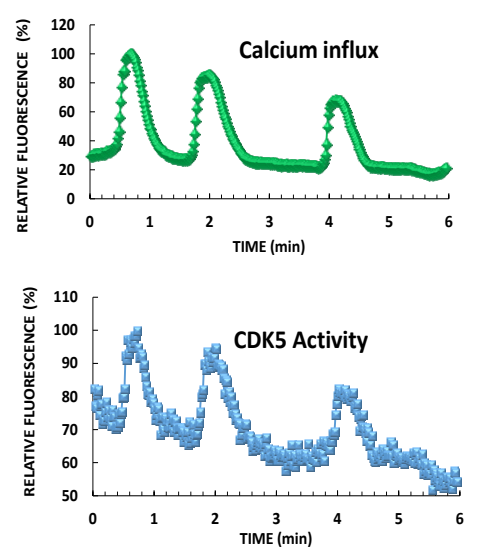

